\title{
Descarga de peso no pós-operatório de fratura de planalto tibial: Revisão sistemática da literatura*
}

\section{Weight Discharge in Postoperative of Plateau Fracture Tibialis: Systematic Review of Literature}

\author{
Débora Pinheiro Lédio Alves ${ }^{10}$ Paloma Yan Lam Wun ${ }^{10}$ Andréia Lima Alves ${ }^{10}$

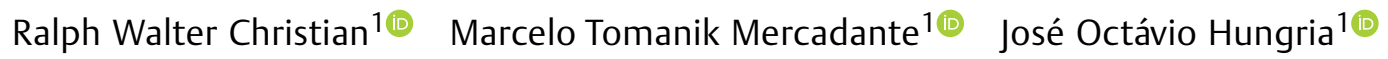 \\ ${ }^{1}$ Departamento de Reabilitação, Faculdade de Ciências Médicas, \\ Santa Casa de Misericórdia de São Paulo, São Paulo, SP, Brasil \\ Endereço para correspondência Andréia de Lima Alves, Rua Doutor \\ Cesário mota Junior, 112, São Paulo, SP, 01221-020, Brasil \\ (e-mail: andreiadelimaalves@gmail.com).
}

Rev Bras Ortop 2020;55(4):404-409.

\section{Resumo \\ Palavras-chave \\ - fraturas da tíbia \\ - suporte de carga \\ - reabilitação \\ - fisioterapia}

As fraturas do planalto tibial constituem risco à integridade funcional do joelho, afetando o alinhamento axial e podendo levar à dor e à incapacidade do indivíduo. $\mathrm{O}$ suporte de carga e a mobilização articular precoce podem prevenir esses déficits e acelerar o processo de reabilitação. O objetivo do presente trabalho foi realizar uma revisão da literatura sobre estudos que citam o início, evolução e critérios de progressão do suporte de carga no pós-operatório das fraturas de planalto tibial. Foram selecionados artigos publicados nos últimos 12 anos, nos idiomas português e inglês, que descrevessem o tempo de início e progressão do suporte de carga, considerando a gravidade da fratura. Foram selecionados 36 artigos na literatura. Não há consenso na literatura quanto ao início e evolução do suporte de carga no pósoperatório das fraturas do planalto tibial. Contudo, observa-se relação entre a gravidade da fratura e o tempo de início da carga.

Tibial plateau fractures are a risk to the functional integrity of the knee, affecting the axial alignment and capable of leading to pain and disability of the individual. Early weight bearing and joint mobilization can prevent these functional deficits. The goal of the present study was to conduct a systematic review of the literature about studies that quote the beginning, evolution, and progression criteria for weight-bearing in the postoperative period of tibial plateau fractures. We selected articles published in the last 12 years, in Portuguese and English, that described the time of onset and progression of weight-bearing, considering the severity of the fracture. Thirty-six articles were selected. There is no consensus in the literature as to the beginning and evolution of weight-bearing in the postoperative period of tibial plateau fractures; however, a relationship between the severity of the fracture and the fixation method has been observed.

\footnotetext{
Trabalho desenvolvido na Santa Casa de Misericórdia de São Paulo, Faculdade de Ciências Médicas, São Paulo, SP, Brasil.
}

recebido

21 de Agosto de 2018 aceito

26 de Fevereiro de 2019
DOI https://doi.org/

$10.1055 / \mathrm{s}-0039-3402454$. ISSN $0102-3616$.
Copyright $(2020$ by Sociedade Brasileira License terms de Ortopedia e Traumatologia. Published by Thieme Revinter Publicações Ltda, Rio de Janeiro, Brazil 


\section{Introdução}

As fraturas do planalto tibial (FPTs) são lesões intra-articulares graves que correspondem a aproximadamente $1 \%$ de todas as fraturas e totalizam $8 \%$ das fraturas que acometem os idosos. Constituem importante risco à integridade funcional do joelho, em especial quando afetam o alinhamento axial, a congruência e/ou a estabilidade articular, podendo resultar em osteoartrose prematura, lesões ligamentares, dor e incapacidade. ${ }^{1}$

A maioria das lesões acometeo côndilo lateral (55-70\%) e está relacionada a traumas de baixa e média energia. As fraturas isoladas do planalto medial correspondem de $10 \mathrm{a}$ $23 \%$ das fraturas articulares proximais da tíbia. As que acometem de maneira complexas o planalto tibial, caracterizadas como fraturas bicondilianas, representam de 10 a $30 \%$, sendo resultado de traumas de alta energia, e estão associadas à grave lesão de tecidos moles. ${ }^{2}$

Este tipo de fratura resulta da aplicação de forças axiais compressivas combinadas com estresses em varo ou em valgo da articulação do joelho e, na maioria dos casos, está relacionada a acidentes automobilísticos e queda ao solo. ${ }^{3} \mathrm{O}$ prognóstico das FPT depende diretamente de cinco fatores: grau de depressão articular, extensão e separação da linha de fratura dos côndilos tibiais, grau de fragmentação, dissociação metafisária, e integridade dos tecidos moles. ${ }^{3,4}$

A classificação de Schatzker é a mais utilizada nas FPTs. ${ }^{3}$ Outro sistema é o proposto pela classificação Arbeitsgemainchaft für Osteosynthesefragen (AO). ${ }^{5} \mathrm{~A}$ - Figura 1 ilustra

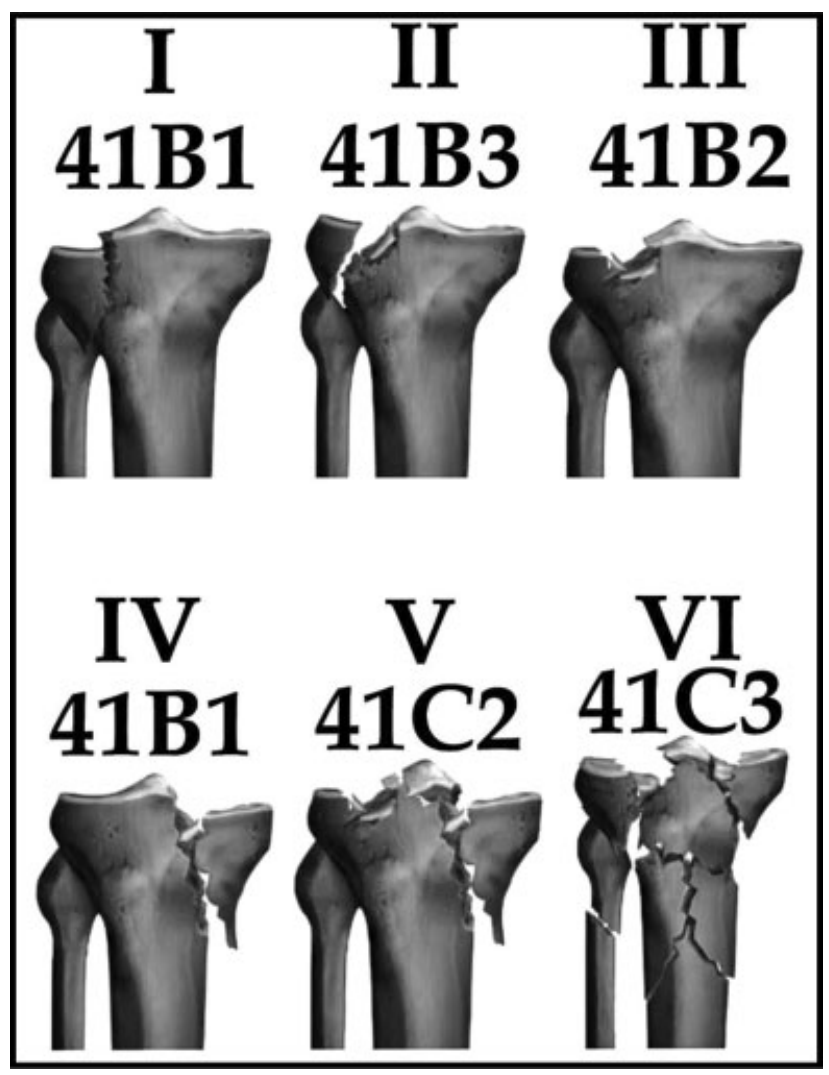

Fig. 1 Correlação entre as classificações de Schatzker e AO. Fonte: Kfuri M, Fogagnolo F, Bitar R, Freitas R, Salim R, Paccola CA. Fraturas do planalto tibial. Rev Bras Ortop. 2009;44(6):468-74. a associação entre essas duas classificações, a de Schatzker e a $\mathrm{AO}^{4}$

O principal objetivo na fixação das FPTs é obter uma articulação estável, congruente e alinhada, preservando a mobilidade articular do joelho e sem dor, permitindo o retorno precoce do paciente às atividades funcionais. ${ }^{6} \mathrm{~A}$ literatura descreve diversos métodos de fixação para fraturas proximais da tíbia, como a redução aberta e fixação interna (RAFI), ${ }^{6,7}$ a redução fechada e fixação externa (RFFE), ${ }^{8,9}$ o parafuso de compressão percutâneo, ${ }^{10}$ o uso de enxerto subcondral, ${ }^{11}$ o uso de substitutos do enxerto (cimento de fosfato de cálcio), ${ }^{12-14}$ e a artroscopia, ${ }^{10,11,15}$ podendo também ocorrer a associação destes métodos. E ainda, para fraturas com desvio mínimo, está indicado o tratamento conservador com uso de órteses articuladas ou braces. $^{16}$

O método cirúrgico de fixação escolhido deve alcançar a redução anatômica dos fragmentos ósseos, promovendo estabilidade absoluta no foco de fratura de forma a criar condições para que ocorra a consolidação direta da fratura. Desta forma, permite mobilização articular e suporte de carga precoce no membro inferior acometido, promovendo a nutrição da cartilagem e prevenindo a formação de artrofibrose no joelho. $6,16,17$

Existem controvérsias na literatura em relação ao período para início e evolução de suporte de carga no membro inferior no pós-operatório das FPTs. Alguns estudos citam períodos variados entre 10 e 12 semanas sem carga, ${ }^{9,17}$ outros relatam 6 a 8 semanas sem carga. ${ }^{10,18}$ Encontram-se também estudos que liberam carga parcial imediata, ${ }^{16,19} \mathrm{e}$ ainda outros relatam que o início de suporte de carga é caso-dependente. ${ }^{20}$

O presente estudo tem como objetivo realizar uma revisão sistemática da literatura a fim de averiguar o tempo de início para o suporte de carga no pós-operatório das FPTs, considerando a gravidade, o método de fixação, e os critérios determinantes para evolução da carga.

\section{Método}

Foi realizada uma revisão sistemática da literatura nas bases de dados eletrônicas EMBASE e PUBMED, relativa aos últimos 18 anos (2000-2018), com o intuito de obter a análise das publicações mais recentes sobre o tema. As buscas foram realizadas a partir do cruzamento dos seguintes descritores de texto, e seus respectivos termos em inglês: "fratura do planalto tibial" ("tibial plateau fracture"), "suporte de carga" ("weight-bearing"), "reabilitação" ("rehabilitation") e "fisioterapia" ("physical therapy").

Os critérios de inclusão adotados foram artigos publicados entre 2000 e 2018, que estivessem nos idioma português e inglês; artigos relacionados à reabilitação no pós-operatório de FPT, independente da sua classificação (Schatzker/AO), podendo apresentar lesões associadas no membro inferior ipslateral (ligamento cruzado anterior, menisco), e que citassem o tempo de liberação do suporte de carga no membro inferior acometido.

Os critérios de exclusão seguidos foram: FPTs tratadas conservadoramente, revisões sistemáticas, estudos experimentais 
em animais, em cadáveres e também em modelos biomecânicos.

Os dados extraídos dos artigos incluídos no presente estudo foram o tempo para início e evolução do suporte de carga, assim como os critérios determinantes para sua progressão. Foram registrados também os métodos de fixação da fratura e suas respectivas classificações.

\section{Resultados}

A partir do cruzamento dos descritores de texto foram identificados 616 artigos nas bases de dados EMBASE, MEDLINE e PUBMED. A dinâmica de inclusão e exclusão dos artigos segue demonstrada na - Tabela 1- ${ }^{6-15,17-28}$ e na - Figura 2.

Ao serem submetidos à primeira análise através da leitura de título e resumo, foram excluídos 569 artigos por não apresentarem temas relacionados à reabilitação no pósoperatório de FPT (comparação entre os métodos de fixação, descrição de técnicas cirúrgicas, tratamento para fraturas que não envolvessem o planalto tibial, tratamento conservador, gonartrose como sequela da fratura), 175 por duplicata, 28 por idioma (17 em alemão, 6 em chinês, 2 em polonês, 1 em japonês, e 3 em turco), 12 por serem estudos laboratoriais em modelos biomecânicos ou cadavéricos e por fim, 4 artigos excluídos por serrem estudos experimentais em animais.

Concluída a primeira análise, restaram 47 artigos selecionados, sendo estes submetidos à segunda análise através da leitura na íntegra do artigo. Destes, 25 foram excluídos, sendo 18 por não apresentarem definição quanto ao início de suporte de carga pós-operatório de FPT, outros eram estudos em forma de revisão bibliográfica que não especificavam a classificação da fratura e o tempo de suporte de carga. Em

Tabela 1 Principais estudos selecionados de acordo com autor/ano de publicação, método de fixação, classificação, início de descarga de peso e progressão de carga

\begin{tabular}{|c|c|c|c|c|c|c|}
\hline Autor & Método Fixação & Schatzker & Toque & Carga parcial & $\begin{array}{l}\text { Carga } \\
\text { total }\end{array}$ & $\begin{array}{l}\text { Critério Evolução } \\
\text { de carga }\end{array}$ \\
\hline Wang et al. ${ }^{25}$ (2016) & Diversos & I, II, III & - & 8 sem. & 12 sem. & \\
\hline Thewlis et al. ${ }^{23}$ (2015) & RAFI (placa) & I, II, III, IV, V & POi & 2 sem. & 12 sem. & Evidência radiológica \\
\hline Benea et al. ${ }^{21}(2015)$ & $\begin{array}{l}\text { RAFI (Redução artroscópica } \\
\text { e fixação interna) }\end{array}$ & I, III & & 8 sem. & 12 sem. & Evidência radiológica \\
\hline Chen et al. ${ }^{7}$ (2015) & RAFI (placa e parafuso) & I, II, III, IV, V & & POi & 12 sem. & Evidência radiológica \\
\hline Giannotti et al. ${ }^{26}(2016)$ & RAFI (placa) & I, II, IV, V, VI & $\ldots$ & 5-6 sem. & $\ldots$ & Conforme o tolerável \\
\hline Spagnolo et al. ${ }^{17}$ (2011) & Fixação Interna (LISS/ZPLT) & VI & & 10-12 sem. & 16 sem. & Calo ósseo metáfise \\
\hline Malakasi et al. ${ }^{6}(2013)$ & RAFI / RFFE híbrido & I, II, III, IV, V, VI & 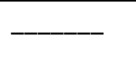 & $\begin{array}{l}\text { RAFI: } 9,8 \mathrm{~s} \text { RFFE } \\
\text { híbrido : } 12,9 \mathrm{~s}\end{array}$ & $\ldots$ & Evidência radiológica \\
\hline Solomon et al. ${ }^{20}(2011)$ & RAFI (placa e parafuso) & ॥ & $\ldots$ & POi & 6 sem. & Evidência radiológica \\
\hline Gupta et al. ${ }^{9}(2010)$ & JESS e Parafuso Percutâneo & $\mathrm{V}, \mathrm{VI}$ & $\ldots$ & 12 sem. & 13-19 sem. & $\begin{array}{l}\text { Evidência Radiológica } \\
\text { (cortical e desvio) }\end{array}$ \\
\hline Mahajan et al. ${ }^{19}$ (2009) & Diversos & I, II, III, IV, V, VI & $\ldots$ & 8 sem. & $\ldots$ & Evidência radiológica \\
\hline Duan et al. ${ }^{18}$ (2008) & RAFI + artroscopia & I, II, III, IV, V & POi (brace) & 8 sem. & $12 \mathrm{sem}$. & $\ldots$ \\
\hline Kayali et al. ${ }^{10}(2008)$ & $\begin{array}{l}\text { Parafuso } \\
\text { percutâneo + artroscopia }\end{array}$ & I, II, III & $\ldots$ & 6 sem. & 10 sem. & Evidência radiológica \\
\hline $\begin{array}{l}\text { Venkatesh et al. }{ }^{14} \\
(2006)\end{array}$ & $\begin{array}{l}\text { Artroscopia e parafusos } \\
\text { percutâneos }\end{array}$ & I,II, III, IV, V, VI & & $\ldots$ & 6 sem. & $\ldots$ \\
\hline Faldini et al. ${ }^{8}$ (2005) & RFFE híbrido & $\mathrm{VI}$ & $\ldots$ & POi. & $8-12$ sem. & Evidência radiológica \\
\hline Ali et al. ${ }^{24}$ (2003) & $\begin{array}{l}\text { RFFE Circular e Parafuso } \\
\text { Percutâneo }\end{array}$ & $\mathrm{V}, \mathrm{VI}$ & POi & 3 sem. & 3-6 sem. & Conforme o tolerável \\
\hline $\begin{array}{l}\text { Horstmann et al. }{ }^{12} \\
\text { (2003) }\end{array}$ & $\begin{array}{l}\text { Cimento de fosfato de cálcio/ } \\
\text { Parafuso Percutâneo/ Placa }\end{array}$ & II, III, VI & PO 2 & 2-6 sem. & 6-12 sem. & Evidência radiológica \\
\hline $\begin{array}{l}\text { Van Glabbeek et al. }{ }^{15} \\
\text { (2002) }\end{array}$ & $\begin{array}{l}\text { Fixação Interna via } \\
\text { artroscópica }\end{array}$ & I, II, IV, V & & 6 sem. & 8 sem. & \\
\hline Larson et al. ${ }^{27}(2002)$ & $\begin{array}{l}\text { Cimento de fosfato de cálcio } \\
\text { e RAFI }\end{array}$ & Revisão & & $\ldots$ & 6 sem. & Evidência radiológica \\
\hline $\begin{array}{l}\text { Lobenhoffer et al. }{ }^{13} \\
(2002)\end{array}$ & $\begin{array}{l}\text { Cimento de fosfato de cálcio } \\
\text { e RAFI }\end{array}$ & I, II, III, VI & & PO 2 & 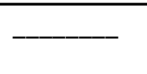 & $\begin{array}{l}\text { I, II, III: } 2 \text { sem. } \\
\text { VI: } 6 \text { sem. }\end{array}$ \\
\hline Kiefer et al. ${ }^{11}$ (2001) & $\begin{array}{l}\text { Artroscópica e enxerto / } \\
\text { parafuso percutâneo/placa }\end{array}$ & I, II, III, VI & $\ldots$ & $\mathrm{POi}$ & 6 sem. & $\ldots$ \\
\hline Sament et al. ${ }^{28}$ (2012) & $\begin{array}{l}\text { Redução fechada e fixação } \\
\text { por parafuso percutaneo }\end{array}$ & I, II, IV, V & $\ldots$ & 6 sem. & 12 sem. & Evidência radiológica \\
\hline Shen et al. ${ }^{22}$ (2009) & $\begin{array}{l}\text { Redução aberta e fixação } \\
\text { interna + B-TCP }\end{array}$ & $\mathrm{II}, \mathrm{V}, \mathrm{VI}$ & & 6a Sem & & Evidência radiológica \\
\hline
\end{tabular}

Legenda: B-TCP, fosfato tricálcico; JESS, Joshi’s external stabilization system; LISS, less invaisve stabilization system; PO 2, pós-operatório 2 dias; POi, pósoperatório imediato; RAFI, redução aberta e fixação interna; RFFE, redução fechada e fixação externa; sem., semanas; ZPLT, Zimmer periarticular locking plate system. 


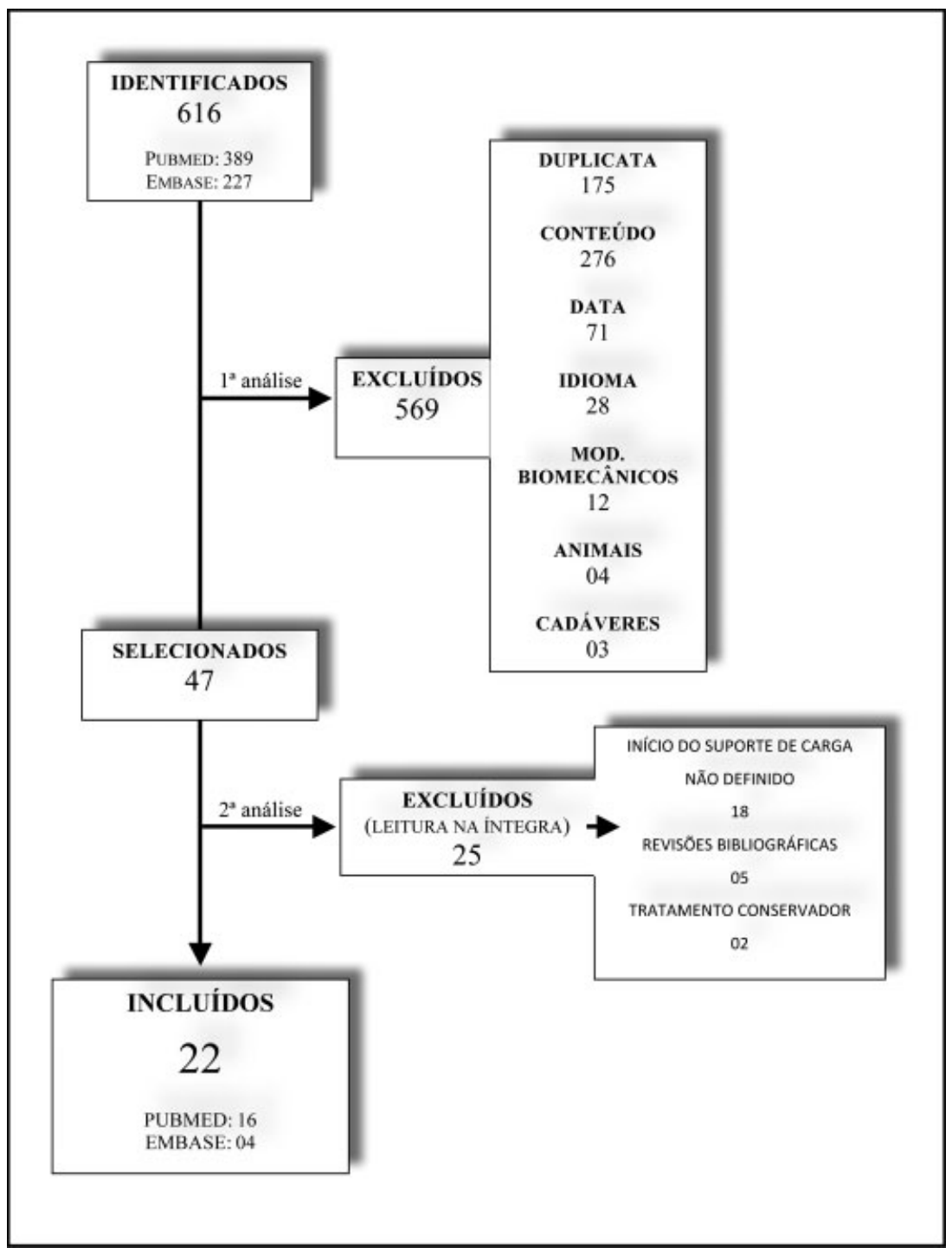

Fig. 2 Fluxograma ilustrando a dinâmica de inclusão e exclusão dos artigos previamente identificados.

dois estudos, a evolução de suporte de carga era descrita apenas em casos tratados conservadoramente.

Portanto, foram incluídos no presente estudo 22 artigos que contemplam os critérios de inclusão previamente determinados.

\section{Discussão}

As FPTs podem evoluir com complicações como instabilidade, desvio axial, incongruência articular, infecção da ferida operatória, e necrose de partes moles. Diversos métodos de fixação são usados para o tratamento dessas fraturas com objetivo de atingir estabilidade absoluta entre os fragmentos e consolidação primária, possibilitando a reabilitação precoce através da mobilização e suporte de carga no membro inferior acometido nos primeiros dias pós-operatório. ${ }^{4,17}$

De acordo com o manual $\mathrm{AO},^{5}$ a liberação de $50 \%$ de suporte de carga deve ocorrer entre 6 e 8 semanas conforme evidências radiográficas independente do tipo da fratura. A progressão da carga em fraturas de baixa energia evolui para carga total progressiva entre 8 e 12 semanas, e no caso de fraturas de alta energia, entre 12 e 16 semanas. $^{5}$

O método de fixação por via artroscópica com parafusos percutâneos e/ou placas de suporte parece obter maior sucesso em fraturas dos tipos I, II, e III de Schatzker, traumas de menor energia. ${ }^{10,11,15,18}$ Essa técnica propicia redução anatômica da superfície articular, permite reparar lesões ligamentares e meniscais e, por ser minimamente invasiva, apresenta também baixas taxas de complicações (tromboembolismo, infecções), menor morbidade, e menor tempo de internação hospitalar, ${ }^{15}$ permitindo assim mobilização articular e deambulação precoce. ${ }^{18,21}$

Quanto à descarga de peso na abordagem artroscópica, van Glabbeek et al. ${ }^{15}$ e Kayali et al. ${ }^{10}$ iniciaram a descarga de peso parcial já na $6^{a}$ semana de pós-operatório, com auxílio do brace articulado, evoluindo para descarga de peso total entre a $8^{\mathrm{a}}$ e a $10^{\mathrm{a}}$ semana de pós-operatório. É importante salientar que quando a classificação de Schatzker foi mais alta ou a fratura mais cominuta os resultados não foram tão satisfatórios com o procedimento artroscópico; ${ }^{15}$ porém, em 
caso de fraturas de baixa energia de Schatzker (I, II e II), a artroscopia é um método alternativo e eficaz. ${ }^{10}$ Kiefer et al., ${ }^{11}$ também em uma abordagem artroscópica, iniciaram a descarga de peso parcial ainda no pós-operatório imediato, e descarga total progressiva a partir da $6^{\mathfrak{a}}$ semana. Segundo os autores, não houve prejuízo quanto a estabilidade e desvio da fratura. Já Duan et al. ${ }^{18}$ liberaram descarga de peso proprioceptiva associada ao uso de um brace articulado a fim de manter o alinhamento e estabilidade da fratura e a evolução da descarga de peso ocorria apenas a partir da $8^{\mathbf{a}}$ semana.

Uma outra alternativa para as fraturas é o uso do cimento de fosfato de cálcio associado ao uso dos parafusos percutâneos e placas de suporte. Horstmann et al. ${ }^{12}$ e Lobenhoffer et al. ${ }^{13}$ abordaram as fraturas Schatzker II, III, e VI com esta técnica e realizaram marcha proprioceptiva no segundo pósoperatório, evoluindo para carga parcial na $2^{\underline{a}}$ semana e descarga de peso total na $6^{\underline{a}}$ semana. Em ambos os estudos, não houve perda de redução da fratura com o cimento de fosfato de cálcio associado a descarga de peso precoce, mostrando-se este método mais resistente que os demais enxertos (osso esponjoso, osso cortical, e a hidroxipatita), tolerando forças torcionais e cargas compressivas. Entretanto, este método apresenta como desvantagem o alto custo de seu material. ${ }^{12,13}$

Existe ainda um estudo que utiliza a cerâmica B-TCP, ou fosfato tricálcico, com a finalidade de transportar fatores de crescimento ou estruturas para as células-tronco mesenquimais, agindo como um osteocondutor reabsorvível. ${ }^{22,29} \mathrm{~A}$ utilização do método tem como objetivo a correção da depressão no planalto tibial para os casos de Schatzker II, V, e VI.S desvantagem é que a liberação da carga parcial inicia-se na $6^{a}$ semana e que a cerâmica B-TCP tem fraca rigidez quando comparada com o cimento de fosfato de cálcio e, segundo o autor, um tempo mais prolongado sem descarga de peso pode contribuir para resultados mais satisfatórios desta técnica. ${ }^{22}$

Outra técnica cirurgica amplamente descrita é a RAFI, que apresenta-se como um método mais invasivo, pois a via de acesso agride tecidos moles e expõe o foco de fratura, tornando-a mais susceptível a infecções. ${ }^{6,19}$ Entretanto, permite a visualização e redução anatômica da fratura e é, muitas vezes, o método mais indicado para as fraturas cominutas. ${ }^{15}$

Uma das questões relacionadas a RAFI é quanto a estabilidade da fratura e o melhor momento para iniciar a descarga de peso. Pensando nisso, Solomon et al. ${ }^{20}$ e Thewlis et al..$^{23}$ realizaram um estudo a fim de quantificar os movimentos dos fragmentos ósseos durante a carga parcial ou sem carga no pós-operatório através da análise radioesterométrica ${ }^{30}$ e em ambos os estudos, observou-se que a migração dos fragmentos da fratura era similar entre os grupos (com e sem carga), mostrando que desvios de até 3,0 $\mathrm{mm}$ não apresentam alterações clínicas com a descarga de peso parcial imediata e que esta daria o estímulo necessário para a consolidação óssea sem interferir no alinhamento e estabilidade da fratura, contribuindo dessa forma para a reabilitação.

No caso de fraturas bicondilianas (Schatzker V e VI), os traumas são de maior energia e costumam apresentar lesão de partes moles importantes. Neste caso, a RFFE híbrida, associada à fixação dos fragmentos articulares com parafusos percutâneos, é a opção. 8,9,24

Algumas técnicas são alternativas para os casos mais graves de fratura e Schatzcker (IV, Ve VI). Uma delas é a técnica Joshi's external stabilization system (JESS), recomendada quando as fraturas são muito cominutas e há grande lesão de partes moles. Essa técnica consiste em uma fixação externa provisória associada à fixação de um parafuso definitivo. ${ }^{9}$ Há ainda a técnica less invasive stabilization system (LISS) com Zimmer periarticular locking plate system (ZPLT), que se trata de uma redução indireta com objetivo de obter redução sem danificar os tecidos moles adjacentes, ou sem operar diretamente no local da fratura, pensando na vascularização periostal e favorecimento do processo de cicatrização. ${ }^{17}$ Uma das limitações de ambas as técnicas é o tempo para início da descarga de peso que ocorreu entre a $10^{\mathrm{a}}$ e $12^{\mathrm{a}}$ segunda semanas.

A RAFI possui vantagem em permitir descarga de peso parcial de forma precoce, conforme desmonstrado nos estudos de Faldini et al. ${ }^{8}$ e Ali et al. ${ }^{24}$ A limitação dessa técnica é a diminuição da precisão da redução da superfície articular. Os resultados obtidos nestes estudos divergem dos resultados do estudo realizado por Malakasi et al., ${ }^{6}$ que comparam a RAFI com a RFFE híbrida para tratamento das fraturas de planalto tibial, mostrando que apesar da RAFI apresentar maior morbidade, ela permite o início da descarga de peso mais precoce do que a RFFE, independente do tipo de Schatzker, tendo uma média de início de carga parcial de 9,8 semanas para RAFI e 12,9 para RFFE.

Levando-se em consideração o tempo de descarga de peso e a gravidade da fratura, observa-se que o início e evolução para o suporte de carga total esta relacionada à gravidade da fratura, ou seja, quanto maior Schatzker mais tempo levará para progredir com o suporte de carga no membro inferior acometido. 6,31

Lobenhoffer et al., ${ }^{13}$ em fraturas Schatzker II, III, e VI, progredia no suporte de carga também conforme tolerado, e assim como outros estudos ${ }^{8,24,32}$ baseavam-se no sistema de pontuação de Rasmussen - ponderando nas radiografias o grau de depressão e largura do planalto, além dos possíveis desvios em valgo/varo do membro inferior. Quanto aos aspectos clínicos, são consideradas a dor, capacidade de deambular, amplitude de movimento, e estabilidade no membro inferior acometido. ${ }^{32}$ E ainda três outros estudos ${ }^{11,12,33}$ não citam seus critérios de evolução do suporte de carga.

Uma limitação importante do presente estudo é que em todos os trabalhos incluidos não havia um consenso entre o tempo ideal para descarga de peso nas fraturas de planalto tibial. Alguns autores já demostraram que a descarga de peso proprioceptiva precoce é indicada e, inclusive, está associada a sucesso na consolidação e reabilitação destes pacientes. $^{20,23}$ Outra limitação do estudo relaciona-se ao tempo de progressão de carga. Alguns estudos defendem que a descarga de peso parcial iniciada na 6 a semana é uma medida eficaz, uma vez que respeita o periodo de consolidação óssea; ${ }^{10,15}$ porém, há autores que defendem que fraturas mais cominutas devem ter uma progressão de carga mais lenta. ${ }^{9,16}$ Além disso, a diversidade de técnicas utilizadas no tratamento das FPTs pode levar a um viés nos resultados, 
visto que há técnicas que favorem o processo de consolidação e descarga de peso precoce ${ }^{12,13}$ e outras que, apesar de demonstrar bons resultados, retardam a liberação da descarga de peso. ${ }^{22}$

\section{Considerações finais}

Não há consenso na literatura quanto ao suporte de carga no pós-operatório de FPT; embora tenha sido observada relação entre a gravidade da fratura, uso de enxerto, tipo de estabilidade e o tempo para início e progressão no suporte de carga. Entretanto, evidenciamos que a maioria dos autores inicia a carga parcial por volta da $6^{\underline{a}}$ semana e carga total na $12^{\underline{a}}$ semana.

Através da presente revisão da literatura, foi possivel observar que há necessidade de novos estudos clínicos randomizados e controlados a respeito da descarga de peso em FPTs nas diversas técnicas cirúrgicas.

\section{Conflito de Interesses}

Os autores declaram não haver conflito de interesses.

\section{Referências}

1 Weigel DP, Marsh JL. High-energy fractures of the tibial plateau. Knee function after longer follow-up. J Bone Joint Surg Am 2002; 84(09):1541-1551

2 Hohl M, Part I. Fractures of the proximal tibia and fibula. In: Rockwood C, Green D, Bucholz R, editors. Fractures in adults. 3rd ed. Philadelphia: Lippincott; 1991

3 Fenton P, Porter K. Tibial plateau fractures: a review. Trauma 2011;13(03):181-187

4 Júnior MK, Fogagnolo F, Bitar RC, Freitas RL, Salim R, Jansen Paccola CA. Tibial plateau fractures. Rev Bras Ortop 2015;44 (06):468-474

5 Ruedi TP, Murphy WM. AO principles of fracture management. Ney York: Thieme Stuttgart; 2000

6 Malakasi A, Lallos SN, Chronopoulos E, Korres DS, Efstathopoulos NE. Comparative study of internal and hybrid external fixation in tibial condylar fractures. Eur J Orthop Surg Traumatol 2013;23 (01):97-103

7 Chen HW, Chen CQ Yi XH. Posterior tibial plateau fracture: a new treatment-oriented classification and surgical management. Int J Clin Exp Med 2015;8(01):472-479

8 Faldini C, Manca M, Pagkrati S, et al. Surgical treatment of complex tibial plateau fractures by closed reduction and external fixation. A review of 32 consecutive cases operated. J Orthop Traumatol 2005;6(04):188-193

9 Zahid M, Sherwani MKA, Siddiqui YS, Abbas M, Asif N, Sabir AB. The role of the JESS (Joshi's external stabilization system) fixator in the management of tibial plateau fractures which are associated with severe soft tissue injuries. J Clin Diagn Res 2010;4:3356-3361

10 Kayali C, Oztürk H, Altay T, Reisoglu A, Agus H. Arthroscopically assisted percutaneous osteosynthesis of lateral tibial plateau fractures. Can J Surg 2008;51(05):378-382

11 Kiefer H, Zivaljevic N, Imbriglia JE. Arthroscopic reduction and internal fixation (ARIF) of lateral tibial plateau fractures. Knee Surg Sports Traumatol Arthrosc 2001;9(03):167-172

12 Horstmann WG, Verheyen CC, Leemans R. An injectable calcium phosphate cement as a bone-graft substitute in the treatment of displaced lateral tibial plateau fractures. Injury 2003;34(02): 141-144
13 Lobenhoffer P, Gerich T, Witte F, Tscherne H. Use of an injectable calcium phosphate bone cement in the treatment of tibial plateau fractures: a prospective study of twenty-six cases with twentymonth mean follow-up. J Orthop Trauma 2002;16(03):143-149

14 Venkatesh R. Minimal invasive techniques in the management of tibial plateau fractures. Curr Orthop 2006;20:411-417

15 van Glabbeek F, van Riet R, Jansen N, D'Anvers J, Nuyts R. Arthroscopically assisted reduction and internal fixation of tibial plateau fractures: report of twenty cases. Acta Orthop Belg 2002; 68(03):258-264

16 Manidakis N, Dosani A, Dimitriou R, Stengel D, Matthews S, Giannoudis P. Tibial plateau fractures: functional outcome and incidence of osteoarthritis in 125 cases. Int Orthop 2010;34(04): $565-570$

17 Spagnolo R, Pace F. Management of the Schatzker VI fractures with lateral locked screw plating. Musculoskelet Surg 2012;96 (02):75-80

18 Duan XJ, Yang L, Guo L, Chen GX, Dai G. Arthroscopically assisted treatment for Schatzker type I-V tibial plateau fractures. Chin J Traumatol 2008;11(05):288-292

19 Mahajan N. Evaluation of results of various operative method in the management of tibial plateau fractures in adults. JK Science. 2009;11(01):27-30

20 Solomon LB, Callary SA, Stevenson AW, McGee MA, Chehade MJ, Howie DW. Weight-bearing-induced displacement and migration over time of fracture fragments following split depression fractures of the lateral tibial plateau: a case series with radiostereometric analysis. J Bone Joint Surg Br 2011;93(06):817-823

21 Benea H, Tomoaia G, Martin A, Bardas C. Arthroscopic management of proximal tibial fractures: technical note and case series presentation. Clujul Med 2015;88(02):233-236

22 Shen C, Ma J, Chen XD, Dai LY. The use of beta-TCP in the surgical treatment of tibial plateau fractures. Knee Surg Sports Traumatol Arthrosc 2009;17(12):1406-1411

23 Thewlis D, Callary SA, Fraysse F, Solomon LB. Peak loading during walking is not associated with fracture migration following tibial plateau fracture: A preliminary case series. J Orthop Res 2015;33 (09):1398-1406

24 Ali AM, Burton M, Hashmi M, Saleh M. Outcome of complex fractures of the tibial plateau treated with a beam-loading ring fixation system. J Bone Joint Surg Br 2003;85(05):691-699

25 Wang Y, Luo C, Zhu Y, et al. Updated Three-Column Concept in surgical treatment for tibial plateau fractures - A prospective cohort study of 287 patients. Injury 2016;47(07):1488-1496

26 Giannotti S, Giovannelli D, Dell'Osso G, et al. Angular stable plates in proximal meta-epiphyseal tibial fractures: study of joint restoration and clinical and functional evaluation. Musculoskelet Surg 2016;100(01):15-18

27 Larsson S, Bauer TW. Use of injectable calcium phosphate cement for fracture fixation: a review. Clin Orthop Relat Res 2002;(395):23-32

28 Sament R, Mayanger JC, Tripathy SK, Sen RK. Closed reduction and percutaneous screw fixation for tibial plateau fractures. J Orthop Surg (Hong Kong) 2012;20(01):37-41

29 LeGeros RZ. Properties of osteoconductive biomaterials: calcium phosphates. Clin Orthop Relat Res 2002;(395):81-98

30 Bottner F, Su E, Nestor B, Azzis B, Sculco TP, Bostrom M. Radiostereometric analysis: the hip. HSS J 2005;1(01):94-99

31 Youdas JW, Kotajarvi BJ, Padgett DJ, Kaufman KR. Partial weightbearing gait using conventional assistive devices. Arch Phys Med Rehabil 2005;86(03):394-398

32 Rasmussen PS. Tibial condylar fractures. Impairment of knee joint stability as an indication for surgical treatment. J Bone Joint Surg Am 1973;55(07):1331-1350

33 Chan YS. Arthroscopy- assisted surgery for tibial plateau fractures. Chang Gung Med J 2011;34(03):239-247 